\title{
Urinary Extracellular Vesicles: Potential Biomarkers of Renal Function in Diabetic Patients
}

\author{
Agnieszka Kamińska, ${ }^{1}$ Mark Platt, ${ }^{2}$ Joanna Kasprzyk, ${ }^{3}$ Beata Kuśnierz-Cabala, \\ Agnieszka Gala-Błądzińska, ${ }^{5}$ Olga Woźnicka, ${ }^{6}$ Benedykt R. Jany, ${ }^{7}$ Franciszek Krok, ${ }^{7}$ \\ Wojciech Piekoszewski, ${ }^{3,8}$ Marek Kuźniewski, ${ }^{9}$ and Ewa L. Stępieńn ${ }^{1}$ \\ ${ }^{1}$ Department of Medical Physics, Marian Smoluchowski Institute of Physics, Faculty of Physics, Astronomy and \\ Applied Computer Science, Jagiellonian University, 30-348 Kraków, Poland \\ ${ }^{2}$ Department of Chemistry, Loughborough University, Loughborough LE11 3TU, UK \\ ${ }^{3}$ Laboratory of High Resolution Mass Spectrometry, Regional Laboratory of Physicochemical Analysis and Structural Research, \\ Faculty of Chemistry, Jagiellonian University, 30-060 Kraków, Poland \\ ${ }^{4}$ Department of Diagnostics, Chair of Clinical Biochemistry, Jagiellonian University Medical College, 31-501 Kraków, Poland \\ ${ }^{5}$ St' Queen Jadwiga Clinical District Hospital No. 2, 35-301 Rzeszów, Poland \\ ${ }^{6}$ Department of Cell Biology and Imaging, Institute of Zoology, Faculty of Biology and Earth Sciences, Jagiellonian University, \\ 30-387 Kraków, Poland \\ ${ }^{7}$ Department of Solid State Physics, Marian Smoluchowski Institute of Physics, Faculty of Physics, Astronomy and \\ Applied Computer Science, Jagiellonian University, 30-348 Kraków, Poland \\ ${ }^{8}$ Department of Analytical Chemistry, Faculty of Chemistry, Jagiellonian University, 30-060 Kraków, Poland \\ ${ }^{9}$ Department of Nephrology, Jagiellonian University Medical College, 31-501 Kraków, Poland
}

Correspondence should be addressed to Ewa Ł. Stępień; e.stepien@uj.edu.pl

Received 28 September 2016; Revised 8 November 2016; Accepted 13 November 2016

Academic Editor: Feng Wang

Copyright ( $) 2016$ Agnieszka Kamińska et al. This is an open access article distributed under the Creative Commons Attribution License, which permits unrestricted use, distribution, and reproduction in any medium, provided the original work is properly cited.

The aim of this study was to check the relationship between the density of urinary EVs, their size distribution, and the progress of early renal damage in type 2 diabetic patients (DMt2). Patients were enrolled to this study, and glycated hemoglobin (HbAlc) below 7\% was a threshold for properly controlled diabetic patients (CD) and poorly controlled diabetic patients (UD). Patients were further divided into two groups: diabetic patients without renal failure (NRF) and with renal failure (RF) according to the Glomerular Filtration Rate. Density and diameter of EVs were determined by Tunable Resistive Pulse Sensing. Additionally, EVs were visualized by means of Transmission and Environmental Scanning Electron Microscopy. Nano-liquid chromatography coupled offline with mass spectrometry (MALDI-TOF-MS/MS) was applied for proteomic analysis. RF had reduced density of EVs compared to NRF. The size distribution study showed that CD had larger EVs (mode) than UD (115 versus $109 \mathrm{~nm} ; p<0.05$ ); nevertheless the mean EVs diameter was smaller in controls than in the CD group (123 versus $134 \mathrm{~nm} ; p<0.05)$. It was demonstrated that EVs are abundant in urine. Albumin, uromodulin, and number of unique proteins related to cell stress and secretion were detected in the EVs fraction. Density and size of urinary EVs reflect deteriorated renal function and can be considered as potential renal damage biomarkers.

\section{Introduction}

Recently, the incidence of diabetes mellitus has grown significantly throughout the world and diabetes becomes the most common cause of kidney injury. It is supposed that about
30 percent of patients with diabetes of type $1(\mathrm{DMt1})$ and 10 to 40 percent of those with type 2 (DMt2) will suffer from renal damage [1-3]. Most of cells release small membrane spherical structures called extracellular vesicles (EVs) which can be classified into three groups: exosomes $(50-100 \mathrm{~nm})$, 
microvesicles $(100-1000 \mathrm{~nm})$, and apoptotic bodies. These vesicles differ in their composition and subcellular origin. EVs can be found in several body fluids, including plasma, urine, saliva, and milk [4]. In particular, urine is a rich reservoir of these vesicles which originate from the cells facing the urinary lumen (epithelial cells). The urinary EVs can reflect the state of the damage of the kidney. Results of several studies indicate that EVs originating from urine have recently emerged as an interesting source of diagnostic disease biomarkers and contain molecules involved in intercellular communication [5-9]. Changes in excretion rates of specific proteins also can have predictive value in the early diagnosis of renal damage [10].

Existing clinical markers such as serum creatinine or urine albumin level are not very sensitive and are generally increased when acute or chronic renal injury is well established [11]. Reliable biomarkers of renal injury are lacking in the renal care. Creatinine measured by laboratories provides little information about the underlying cause of renal injuries and is less accurate for patients with low muscle mass $[12,13]$. In diabetes, the most serious and life treating complication is diabetic nephropathy. To avoid this end stage complication there is a growing need to discover novel noninvasive biomarkers of primary renal damage which allow detecting changes in kidney at early stage [14]. In the present study we test the hypothesis that the density and size of urinary EVs can be considered as biomarkers of renal damage in DMt2 patients.

The motivation of this study was to demonstrate the potential usefulness of urinary EVs in diagnostics of early renal failure as a complication of diabetes. In order to achieve this goal we applied the modern approach for urine analysis: Tunable Resistive Pulse Sensing (TRPS) for EVs enumeration and size distribution analysis, a nano-liquid chromatography technique coupled offline with mass spectrometry (MALDI-TOF-MS/MS) for proteomic analysis and electron microscopy (Transmission Electron Microscopy (TEM); Environmental Scanning Electron Microscopy (ESEM)) for EVs visualization.

\section{Materials and Methods}

2.1. Study Group. Sixty patients (20 women and 40 men) with type 2 diabetes mellitus (DMt2) were enrolled to the present study. These patients were divided into groups: CD, properly controlled $(n=24)$, and UD, poorly controlled diabetes $(n=36)$. As a control, ten healthy subjects ( 4 women and 6 men) with an average age of $52(\mathrm{SD}=7)$ years were included. The studied groups were allocated according to the criterion of glycated hemoglobin (HbAlc) levels. According to Polish Diabetes Association guidelines from 2014, a HbAlc level of $7 \%$ is general criterion of carbohydrate metabolism compensation. Patients in whom HbAlc levels exceed 7\% are considered as they have poorly controlled diabetes. What is more, diabetic patients were further classified into two groups: diabetic patients without renal failure (NRF) and with renal failure (RF). A selection of RF was Glomerular Filtration Rate (GFR) below $60 \mathrm{~mL} / \mathrm{min} / 1.73 \mathrm{~m}^{2}$ from MDRD2 formula. Microalbuminuria was defined as $20-200 \mathrm{mg} / \mathrm{L}$ and macroalbuminuria $>200 \mathrm{mg} / \mathrm{L}$ albumin filtration. The clinical characteristics of the studied groups are presented in Tables 1 and 2.

2.2. Urine Samples Collection and Preparation. First morning urine specimens were collected into sterile containers (F.L. Medical SRL, Torreglia, Italy) from diabetic patients and healthy subjects. Typically $50 \mathrm{~mL}$ first void urine was used for the isolation of the urinary extracellular vesicles and processed within $2 \mathrm{~h}$ of collection. Samples were centrifuged in a Hermle Z300K (Hermle Labortechnik GmbH, Wehingem, Germany) for $10 \mathrm{~min}$ in $3000 \mathrm{~g}$ at $4^{\circ} \mathrm{C}$ to remove cells and larger debris. After this step supernatants were aliquoted and frozen at $-80^{\circ} \mathrm{C}$ for further analysis. Immediately before the TRPS measurement, samples were thawed in a water bath at $37^{\circ} \mathrm{C}$ and then vortexed for $30 \mathrm{~s}$, diluted $1: 1$ in PBS (Cat. number P4417, Sigma-Aldrich, St. Louis, USA), vortexed for $10 \mathrm{~s}$, and used for analysis. For mass spectrometry and electron microscopy analysis, supernatants were ultracentrifuged in $150000 \mathrm{~g}$ for $1 \mathrm{~h}$ at $4^{\circ} \mathrm{C}$ (Optima ${ }^{\mathrm{TM}}$ MAX-XP, Beckman Coulter Life Sciences, Indianapolis, USA) using a horizontal rotor (Cat. number 367280, MLS-50 Swinging-Bucked Rotor, Beckman Coulter Life Sciences, Indianapolis, USA).

2.3. Blood Samples. Blood samples for biochemical and hematology analysis were drawn by venipuncture of the antecubital vein using a 21-gauge needle and the Sarstedt S-Monovette blood collection system (Sarstedt AG \& Co., Nümbrecht, Germany) following application of a light tourniquet. For complete blood count analysis and HbAlc levels, EDTA anticoagulant was used. For biochemical analysis, blood was collected in serum separator tubes. Standard blood tests were performed by means of the hematology analyzer (ELITech Group, Puteaux, France). HbAlc level was measured on D-10 analyzer (D-10 hemoglobin testing system, Bio-Rad Laboratories Inc., California, USA).

2.4. Tunable Resistive Pulse Sensing Technology. The size and density of urinary EVs were determined by Tunable Resistive Pulse Sensing (TRPS) technique using qNano system and tunable pore specimen, NP150 from Izon Science (Izon Science Ltd., Christchurch, New Zealand). Principles of the technique were described in [15-18]. To detect particles in the range $60-480 \mathrm{~nm}$ the pores labeled NP150 were used. Polystyrene beads of known raw concentration $(1.5 E+$ $13 / \mathrm{mL}$ ) and diameter of $105 \mathrm{~nm}$ were sourced from Izon Science and were used as a calibrant. Typically a bandwidth filter of $5 \mathrm{kHz}$ was applied during measurements. For the electrolyte and dilution buffer we used PBS. In all measurements $75 \mu \mathrm{L}$ of electrolyte buffer was placed in the lower fluid cell and the volume in the upper fluid cell was $40 \mu \mathrm{L}$. Each sample was measured in triplicate. The density, mean, and mode diameter of EVs are expressed as median (IQR). Data capture was performed using Izon's control suite 3.1 software.

\subsection{Proteomics (Nano-LC-MALDI-TOF/TOF Mass Spectrom-} etry). For proteomics analysis urinary EVs were isolated from microalbuminuric (CD) and macroalbuminuric (UD) patients and healthy subjects, at least $n=3$ from each 
TABLE 1: Clinical characteristics, blood, and urine biochemistry of study groups: C, CD, and UD.

\begin{tabular}{|c|c|c|c|c|}
\hline & $\begin{array}{c}\mathrm{C} \\
n=10\end{array}$ & $\begin{array}{c}\mathrm{CD} \\
n=24\end{array}$ & $\begin{array}{c}\mathrm{UD} \\
n=36\end{array}$ & $p$ value \\
\hline $\begin{array}{l}\text { Age } \\
\text { (years) }\end{array}$ & $52(7)$ & $62(15)^{\dagger}$ & $61(12)^{\dagger}$ & 0.0683 \\
\hline $\begin{array}{l}\text { Gender } \\
\text { (male/female) }\end{array}$ & $6 / 4$ & $17 / 7$ & $23 / 13$ & - \\
\hline Serum glucose & 5.2 & $6.8^{\dagger *}$ & $9^{\dagger *}$ & \multirow{2}{*}{$<0.0001$} \\
\hline$(\mathrm{mmol} / \mathrm{L})$ & $(5.0-5.5)$ & $(5.9-7.9)$ & $(7.4-12)$ & \\
\hline Urine albumin & 6 & $6^{*}$ & $37^{\dagger *}$ & \multirow{2}{*}{$<0.0001$} \\
\hline$(\mathrm{mg} / \mathrm{L})$ & $(4-13)$ & $(2-22)$ & $(12-267)$ & \\
\hline Urine creatinine & 15 & $5^{\dagger}$ & $7^{\dagger}$ & \multirow{2}{*}{0.0054} \\
\hline$(\mathrm{mmol} / \mathrm{L})$ & $(9-17)$ & $(4-9)$ & $(5-11)$ & \\
\hline $\begin{array}{l}\text { Serum creatinine } \\
(\mu \mathrm{mol} / \mathrm{L})\end{array}$ & $\begin{array}{c}72 \\
(60-85)\end{array}$ & $\begin{array}{c}77 \\
(67-98)\end{array}$ & $\begin{array}{c}79 \\
(62-108)\end{array}$ & 0.4696 \\
\hline $\begin{array}{l}\text { GFR } \\
\left(\mathrm{mL} / \mathrm{min} / 1.73 \mathrm{~m}^{2}\right)\end{array}$ & $\begin{array}{c}87 \\
(76-101)\end{array}$ & $\begin{array}{c}77 \\
(59-95)\end{array}$ & $\begin{array}{c}79 \\
(59-97)\end{array}$ & 0.5114 \\
\hline EVs density & $5.2 E 10$ & $8.4 E 10$ & $5.2 E 10$ & \multirow[t]{2}{*}{0.5013} \\
\hline (number/mL) & $(2.7 E 10-1.9 E 11)$ & $(3.9 E 10-1.7 E 11)$ & $(2.6 E 10-1.5 E 11)$ & \\
\hline EVs mode diameter & 106 & $115^{\dagger *}$ & $109^{*}$ & \multirow{2}{*}{0.0212} \\
\hline$(\mathrm{nm})$ & $(104-110)$ & $(107-118)$ & $(106-112)$ & \\
\hline $\begin{array}{l}\text { EVs mean diameter } \\
(\mathrm{nm})\end{array}$ & $123(4)$ & $134(11)^{\dagger}$ & $129(8)$ & 0.0065 \\
\hline
\end{tabular}

${ }^{\dagger}$ Significant in comparison with the control group at $p<0.05$.

* Significant difference between subgroups CD and UD at $p<0.05$.

Bold means statistically significant difference between the three groups at $p<0.05$.

TABLE 2: Clinical characteristics, blood, and urine biochemistry of study groups: C, RF, and NRF.

\begin{tabular}{|c|c|c|c|c|}
\hline & $\begin{array}{c}\mathrm{C} \\
n=10\end{array}$ & $\begin{array}{c}\mathrm{RF} \\
N=15\end{array}$ & $\begin{array}{c}\text { NRF } \\
N=45\end{array}$ & $p$ value \\
\hline $\begin{array}{l}\text { Age } \\
\text { (years) }\end{array}$ & $52(7)$ & $69(11)^{\dagger *}$ & $60(3)^{\dagger *}$ & 0.0027 \\
\hline $\begin{array}{l}\text { Gender } \\
\text { (male/female) }\end{array}$ & $6 / 4$ & $15 / 3$ & $25 / 17$ & - \\
\hline Serum glucose & 5.2 & $8.7^{\dagger}$ & $7.9^{\dagger}$ & \multirow[t]{2}{*}{$<0.0001$} \\
\hline$(\mathrm{mmol} / \mathrm{L})$ & $(5.0-5.5)$ & $(6.5-11)$ & $(6.5-10)$ & \\
\hline Urine albumin & $6^{\dagger}$ & $51^{\dagger}$ & 14 & \multirow{2}{*}{0.0923} \\
\hline$(\mathrm{mg} / \mathrm{L})$ & $(4-13)$ & $(7-359)$ & $(4-58)$ & \\
\hline Urine creatinine & 15 & $6^{\dagger}$ & $8^{\dagger}$ & \multirow{2}{*}{0.0046} \\
\hline$(\mathrm{mmol} / \mathrm{L})$ & $(9-17)$ & $(5-8)$ & $(4-11)$ & \\
\hline Serum creatinine & 72 & $119^{\dagger *}$ & $73^{*}$ & \multirow{2}{*}{$<0.0001$} \\
\hline$(\mu \mathrm{mol} / \mathrm{L})$ & $(60-85)$ & (111-123) & $(60-84)$ & \\
\hline GFR & 87 & $49^{\dagger *}$ & $89^{*}$ & \multirow{2}{*}{$<0.0001$} \\
\hline$\left(\mathrm{mL} / \mathrm{min} / 1.73 \mathrm{~m}^{2}\right)$ & $(76-101)$ & $(39-55)$ & $(73-105)$ & \\
\hline EVs density & $5.2 E 10$ & $2.6 E 10^{*}$ & $8.7 E 10^{*}$ & \multirow{2}{*}{0.0361} \\
\hline (number/mL) & $(2.7 E 10-1.9 E 11)$ & $(2.0 E 10-8.2 E 10)$ & $(4.0 E 10-1.9 E 11)$ & \\
\hline $\begin{array}{l}\text { EVs mode diameter } \\
(\mathrm{nm})\end{array}$ & $\begin{array}{c}106 \\
(104-110)\end{array}$ & $\begin{array}{c}111 \\
(105-115)\end{array}$ & $\begin{array}{c}109 \\
(107-115)\end{array}$ & 0.1965 \\
\hline EVs mean diameter & 122 & 129 & $129^{\dagger}$ & \multirow{2}{*}{0.0101} \\
\hline$(\mathrm{nm})$ & $(120-126)$ & $(123-138)$ & $(126-136)$ & \\
\hline
\end{tabular}

${ }^{\dagger}$ Significant in comparison with the control group at $p<0.05$.

${ }^{*}$ Significant difference between subgroups RF and NRF at $p<0.05$.

Bold means statistically significant difference between the three groups at $p<0.05$. 
group. After ultracentrifugation, urine supernatants $(6 \mathrm{~mL})$ were used for analysis. Obtained pellet was resuspended in $60 \mu \mathrm{L} \mathrm{10 \%} \mathrm{SDS} \mathrm{(Cat.} \mathrm{number} \mathrm{L3771,} \mathrm{Sigma-Aldrich,} \mathrm{St.}$ Louis, USA), $10 \mu \mathrm{L} 1 \mathrm{M}$ TRIS (Cat. number T1503 SigmaAldrich, St. Louis, USA), and $30 \mu \mathrm{L}$ deionized water [19]. Protein concentration was determined using BCA method (Cat. number 23227, Pierce Biotechnology, Thermo Scientific, USA). Mean protein concentration was $1.14 \pm 1.04 \mathrm{mg} / \mathrm{mL}$; the total protein amount used for MS was $40 \mu \mathrm{g}$. Proteomic analysis was performed by means of a nano-liquid chromatograph (EASY-nLC II ${ }^{\mathrm{TM}}$, Bruker Daltonics, Germany). The detailed methodology was previously published [20]. The precision tolerance was $100 \mathrm{ppm}$ for peptide masses and $0.7 \mathrm{Da}$ for fragment ion masses. Individual peptide matches with scores above 28 were considered statistically significant. Proteins identification was performed manually, based on two unique peptides with the probability less than 0.05 . The protein classification was performed by means of a free algorithm applied in the PANTHER Classification System (Version 11.0, released July 15, 2016) [21]. The analysis of overlapping proteins within healthy subjects, $\mathrm{CD}$, and UD was performed by a tree-circle Venn diagram software [22].

\subsection{Transmission Electron Microscopy and Environmental Scanning Electron Microscopy}

2.6.1. ESEM. Urine sample from a healthy donor $(100 \mathrm{~mL})$ was centrifuged in $3000 \mathrm{~g}$ and next supernatant was ultracentrifuged in $150000 \mathrm{~g}$ for $1 \mathrm{~h}$ at $4^{\circ} \mathrm{C}$. EVs pellet was resuspended in $60 \mu \mathrm{L}$ of PBS and $20 \mu \mathrm{L}$ of EVs solution was placed on $1 \times 1 \mathrm{~cm}$ poly-l-lysine slide (Cat. number J2800 AMNZ, Thermo Fisher Scientific, Waltham, Massachusetts, USA) and incubated for $1 \mathrm{~h}$ in humid chamber at RT. After incubation the slide was washed twice in PBS and fixed in 3.7\% glutaraldehyde in PBS for 30 min followed by salt removal stage. The slide with EVs was washed with two aqueous PBS dilutions, 50\% PBS, 25\% PBS, and deionized water, each for 1 minute. Next, the dehydration was applied by immersing sample for 30 seconds in ethyl alcohol solutions as follows: $10 \%, 20 \%, 30 \%, 40 \%, 50 \%, 60 \%, 70 \%, 80 \%, 90 \%$, and absolute ethanol. Afterwards, sample was dried for $24 \mathrm{~h}$ under cover at RT [23].

The Environmental Scanning Electron Microscopy (ESEM) measurements were performed using SEM Quanta 3D FEG microscope by FEI Company (USA) operated at Institute of Physics Jagiellonian University, Kraków, Poland. The ESEM images were collected by GSED detector using electrons of $5 \mathrm{keV}$ energy. During measurements the specimen was kept at $100 \mathrm{~Pa}$ of water vapor at RT.

2.6.2. TEM. Two urine samples from a healthy donor and one UD were prepared in the same way as for ESEM analysis. Samples were centrifuged in Eppendorf tube and fixed with 2.5\% glutaraldehyde (Cat. number G5882, Sigma-Aldrich, St. Louis, USA) in 0.1M cacodylic buffer (Cat. number C4945, Aldrich, St. Louis, USA) for $2 \mathrm{~h}$ at RT and then postfixed in $1 \%$ osmium tetroxide solution (1 hour). Samples were dehydrated by passing through a graded ethanol series and embedded in PolyBed 812 at $68^{\circ} \mathrm{C}$.
Ultrathin sections were collected on 300 mesh grids or one slot made from copper. Additionally the latter was covered with formvar film. Next the sections were contrasted using uranyl acetate and lead citrate. For observation the electron microscopy from JEOL company JEOL JEM 2100HT (Jeol Ltd, Tokyo, Japan) was used at accelerating voltage $80 \mathrm{kV}$.

2.7. CD81 TRIFIc Exosome Assay. Europium Time Resolved Fluorescence assay, Cat. number EX103 (Cell Guidance Systems Ltd., Cambridge, United Kingdom), was used to measure abundance of human CD81 protein in the surface of exosomes in the same urine samples. In the TRIFIc exosome assay the same antibody is used for binding of target to the assay plate and for detection. This assay consists of a monoclonal antibody (labeled with biotin) bound to streptavidin coated plate that captures proteins which are present in the surface of exosomes. An identical monoclonal antibody (labeled with Europium) is used for detection. Europium provides a high degree of sensitivity for the assay. For fluorescence detection we used infinite M200 PRO plate reader (Tecan Group Ltd., Männedorf, Switzerland).

2.8. Statistical Analysis. Statistica 12 (Dell Statistica, Tulsa, USA) and OriginPro 2016 (OriginLab Corporation, Northampton, USA) were used for statistical analyses and plots design. The distribution of continuous data was verified with Shapiro-Wilk normality test. Results are expressed as mean (SD) for data with normal distribution or median and interquartile ranges (Q1-Q3) for data with not normal distribution. Biochemical and epidemiological data were analyzed by one-way analysis of variance ANOVA or Kruskal-Wallis for comparison among groups. Differences between subgroups were tested with Tukey's post hoc test or Dunn's multiple comparison test. The Mann-Whitney $U$ test was used to compare differences between two independent groups. Correlations between EVs density and biochemical parameters were calculated with Spearman's rank correlation test, and multiple regression (backward stepwise regression) was performed to predict the effect of age on the other variables. For all analyses $p$ values $<0.05$ were considered significant.

2.9. Ethical Considerations. This study was approved by The Bioethical Committee of Jagiellonian University in Kraków on 24 October 2013 which accepted all project's protocols and forms, including an information for patients form and a consent form. The permission number KBET/206/B/2013 is valid until 31 December 2016.

\section{Results}

A comparison of biochemical parameters such as serum glucose, urine albumin, urine creatinine, serum creatinine, GFR, EVs density, EVs mode, and mean diameter in CD, UD, and the control group was provided in Table 1. Properly controlled diabetic patients $(C D)$ and poorly controlled diabetic patients (UD) had significantly higher levels of serum glucose (6.8 versus $5.2 \mathrm{mmol} / \mathrm{L} ; p<0.0001$ and 9 versus $5.2 \mathrm{mmol} / \mathrm{L} ; p<$ 
TABLE 3: Results of Spearman's rho test for correlations between EVs density and biochemical parameters.

\begin{tabular}{lccccc}
\hline & $\mathrm{C}$ & $\mathrm{CD}$ & $\mathrm{UD}$ & $\mathrm{RF}$ & $\mathrm{NRF}$ \\
& $n=10$ & $n=24$ & $n=36$ & $n=15$ & $n=45$ \\
\hline Serum glucose & 0.49 & -0.27 & -0.33 & $\mathbf{- 0 . 6 6}$ & -0.21 \\
(mmol/L) & $p=0.15$ & $p=0.19$ & $p=0.05$ & $\mathbf{p}=\mathbf{0 . 0 1}$ & $p=0.16$ \\
Urine creatinine & 0.08 & $\mathbf{0 . 5 2}$ & 0.03 & -0.46 & $\mathbf{0 . 3 3}$ \\
(mmol/L) & $p=0.83$ & $\mathbf{p}=\mathbf{0 . 0 1}$ & $p=0.87$ & $p=0.08$ & $\mathbf{p}=\mathbf{0 . 0 3}$ \\
Urine albumin & -0.16 & 0.37 & 0.14 & -0.03 & 0.25 \\
$(\mathrm{mg} / \mathrm{L})$ & $p=0.65$ & $p=0.08$ & $p=0.42$ & $p=0.92$ & $p=0.09$ \\
GFR & 0.50 & 0.26 & 0.27 & $-\mathbf{0 . 5 4}$ & 0.07 \\
$\left(\mathrm{~mL} / \mathrm{min} / 1.73 \mathrm{~m}^{2}\right)$ & $p=0.14$ & $p=0.21$ & $p=0.12$ & $\mathbf{p}=\mathbf{0 . 0 4}$ & $p=0.66$ \\
\hline
\end{tabular}

Bold means statistically significant correlation at $p<0.05$ level.

0.0001 ) and lower urine creatinine concentration (5 versus $12 \mathrm{mmol} / \mathrm{L} ; p=0.003$ and 7 versus $12 \mathrm{mmol} / \mathrm{L} ; p=0.004$ ) in comparison with the control group.

Our results showed statistically significant difference in serum glucose $(6.8$ versus $9 \mathrm{mmol} / \mathrm{L} ; p=0.0001)$ and urine albumin ( 6 versus $37 \mathrm{mg} / \mathrm{L} ; p=0.002$ ) between $\mathrm{CD}$ and UD groups. No significant difference was found in serum creatinine concentration, GFR and EVs density between these groups. Size distribution analysis showed that CD had significantly larger EVs mode diameter besides UD (115 versus $109 \mathrm{~nm} ; p=0.031$ ). The mean EVs diameter was smaller in controls than in the CD group (123 versus $134 \mathrm{~nm}$; $p=0.004)$.

A comparison of biochemical parameters in RF, NRF, and the control group is provided in Table 2. Compared with the control group, RF had significantly higher levels of serum glucose (8.7 versus $5.2 \mathrm{mmol} / \mathrm{L} ; p<0.0001)$ and serum creatinine (119 versus $72 \mu \mathrm{mol} / \mathrm{L} ; p<0.0001$ ) and lower urine creatinine concentration ( 6 versus $15 \mathrm{mmol} / \mathrm{L} ; p=$ 0.002 ) and GFR (49 versus $87 \mathrm{~mL} / \mathrm{min} / 1.73 \mathrm{~m}^{2} ; p<0.0001$ ). NRF had significantly higher levels of serum glucose (7.9 versus $5.2 \mathrm{mmol} / \mathrm{L} ; p<0.0001)$ and lower urine creatinine concentration ( 8 versus $15 \mathrm{mmol} / \mathrm{L} ; p=0.003$ ) in comparison with the healthy subjects.

The obtained results indicate that RF had significantly reduced density of EVs compared to NRF (2.57E10 versus $8.73 E 10$ number $/ \mathrm{mL} ; p=0.017)$. We observed statistically significant difference in serum creatinine (119 versus 73 $\mu \mathrm{mol} / \mathrm{L} ; p<0.0001$ ) and GFR (49 versus $89 \mathrm{~mL} / \mathrm{min} / 1.73 \mathrm{~m}^{2}$; $p<0.0001)$ between RF and NRF groups and in albumin level between RF and healthy subjects (51 versus $6.2 \mathrm{mg} / \mathrm{L}$; $p=0.02)$. Because of high variability within patients groups, no significant difference was found between EVs mode diameters between RF and NRF.

Results of Spearman's rho test for relationship between EVs density and biochemical parameters are presented in Table 3. We observed a negative tendency between EVs density and serum glucose level in UD $(R=-0.33)$ and negative correlation in RF $(R=-0.66)$ patients (Figure 2$)$. There was no correlation between these parameters in CD $(p=0.19)$ and NRF $(p=0.16)$. We found positive relationship between EVs density and urine creatinine concentration in CD $(R=$
$0.52)$ and NRF $(R=0.33)$ (Figure 3$)$. There was no correlation between these parameters in UD $(p=0.87)$ and $\mathrm{RF}(p=$ $0.08)$.

Taking into consideration that the age can influence renal function, multiple regression (backward stepwise regression) was performed to show the impact of age on changes in the amount of EVs (see Supplementary Table 1 in the Supplementary Material available online at http://dx.doi.org/10.1155/2016/5741518). Additionally, the correlations of specific biochemical parameters (creatinine, albumin, serum glucose, etc.) with age have been analyzed. Not surprisingly, there was no correlation in control group. The age related negative relationship was observed in CD and NRF group in terms of creatinine clearance (GFR). Such relationship was less significant in patients with more advanced stage of disease (Supplementary Table 2).

Environmental Scanning Electron Microscopy (ESEM) confirmed the presence of the EVs in pellets sedimented after ultracentrifugation of collected samples (Figures 1(a)-1(d)). Washed EVs formed clustered aggregates, which were better distinguishable by means of TEM (Figures 1(e) and 1(f)). The size of EVs was estimated in the range of 130-160 nm. However, a number of smaller and bigger vesicles and other objects were observed.

In order to see the origin and biological activity of analyzed EVs, the proteomic analysis of a urinary EVs fraction was performed. Despite the fact that urine samples were obtained from patients in different stage of DM and different albuminuria levels, the albumin was the main and most abundant protein detected using mass spectrometry methods (Figures 4(a) and 4(b)). The second abundant protein in urine was uromodulin. Venn analysis shows the possible relationships in a protein profile between CD and UD compared to a control subject. Among total 92 proteins in $\mathrm{CD}, 49$ were unique and 31 were common in $\mathrm{CD}$ and UD (Supplementary Table 1). In the UD sample, the total number of proteins was 45 while in the control sample 17 proteins were found. The list of unique proteins for every group was listed in Supplementary Table2. For prediction of common protein interactions the list of common 45 proteins was analyzed by means of Search Tool for the Retrieval of Interacting Genes/Proteins (STRING) [24] (Figure 4(b)). 


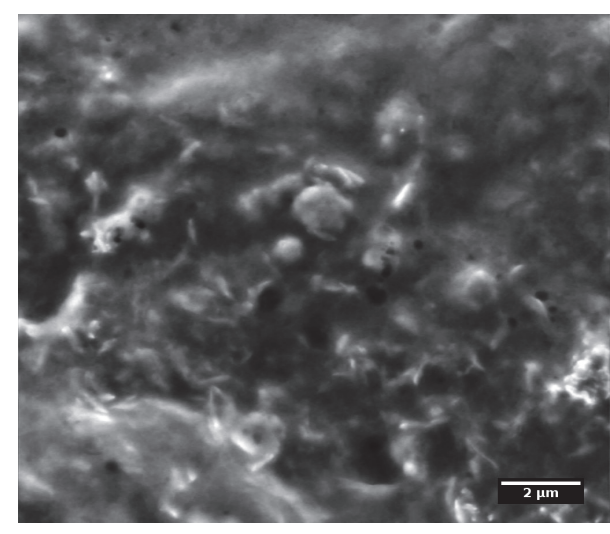

(a)

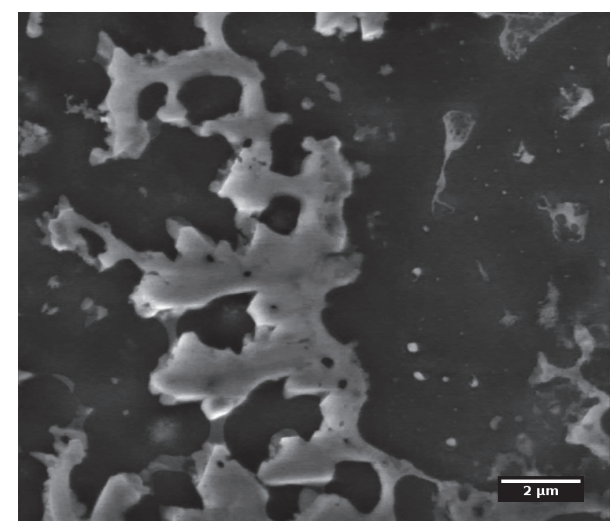

(c)

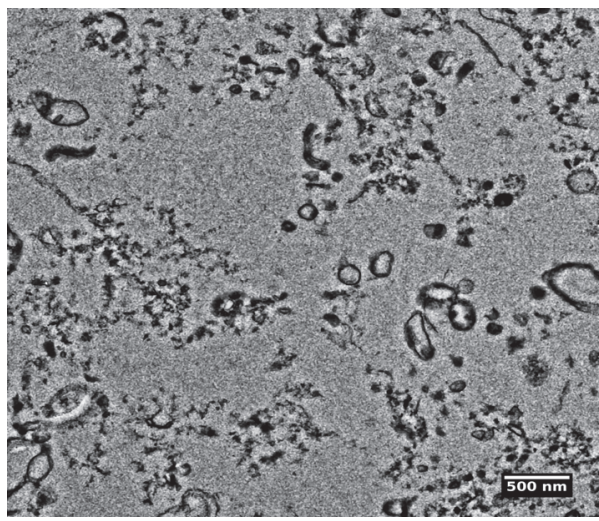

(e)

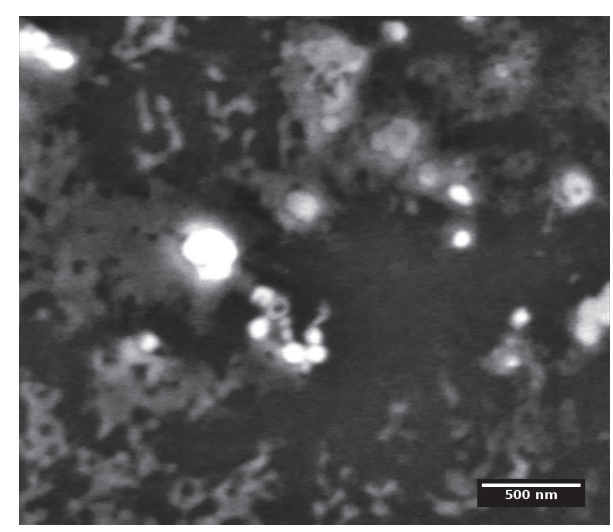

(b)

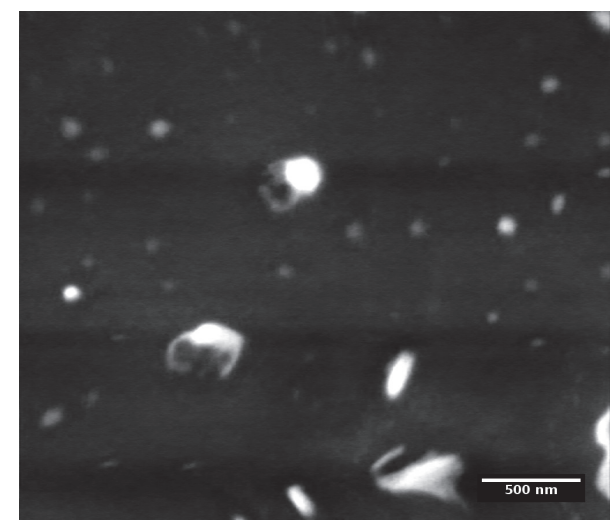

(d)

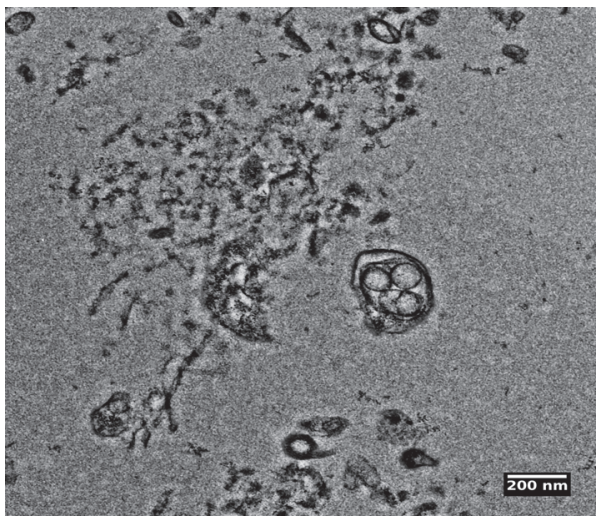

(f)

Figure 1: Environmental Scanning Electron Microscopy (ESEM) (a-d) and Transmission Electron Microscopy (e, f) images of urinary extracellular vesicles (EVs) isolated from a urine sample. ESEM images show that EVs form aggregates and they are clustered on the surface. TEM analysis visualizes the variety of different vesicle-like objects in diameter mostly around 130-160 nm. Interestingly, multivesicle objects were also present in urine that confirms integrity of EVs during preparation.

This analysis revealed the central role of albumin in EVs fraction, nevertheless stress related proteins (ceruloplasmin, transferrin) and cellular components (mostly exosome and extracellular region) (Figures 4(c) and 4(d)).

CD81 TRIFIc exosome assay has not shown any statistical significant differences in CD81 level between study groups, what is presented in Figure 5.

\section{Discussion}

To date, there are no noninvasive methods to characterize renal structural pathophysiological changes [25]. Moreover, biochemical markers are not sensitive enough to characterize the risk of progression of nephropathy and other DM-related complications [13]. 


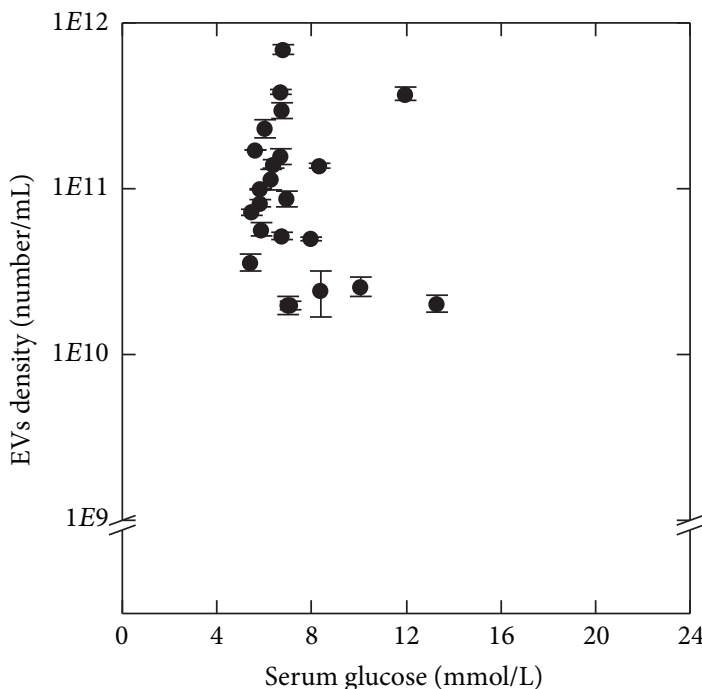

(a)

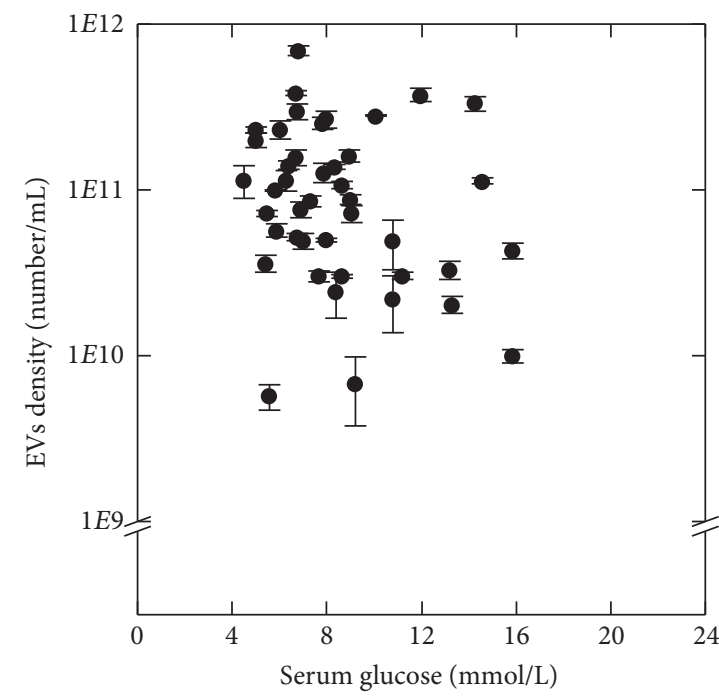

(c)

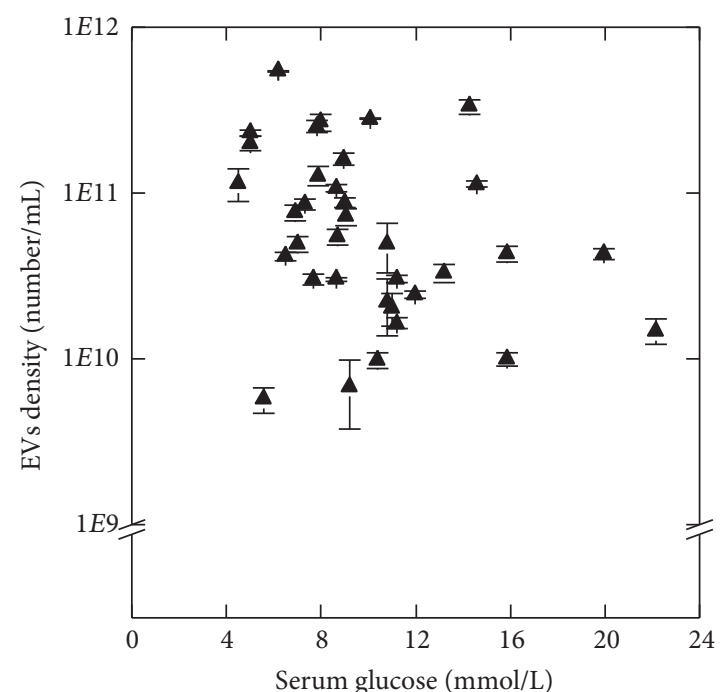

(b)

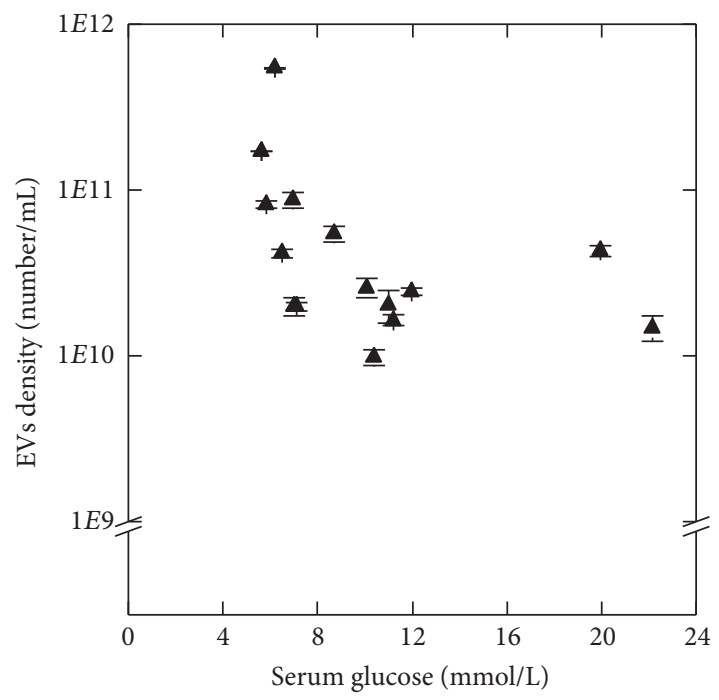

(d)

Figure 2: Relationship between EVs density and serum glucose level in study groups: CD (a), UD (b), NRF (c), and RF (d). EVs density values are given as mean (SD). Spearman's rank correlation coefficient, $p<0.05$.

In this study we sought to test if the density and size of urinary EVs can be considered as potential biomarkers of early renal damage in DMt2 patients which can lead to diabetic nephropathy. Additionally, we studied if there is any correlation between EVs density and biochemical parameters in diabetic patients and healthy control group.

Our results indicate that diabetic patients with renal failure (RF) had lower density of EVs compared to diabetic patients without renal failure (NRF). The size distribution study showed significant difference in EVs mode diameter between CD and UD. Turco et al. [26] showed that decreases of EVs may reflect atherosclerosis and thrombosis-related activity in renal capillaries and parenchyma.

Currently kidney function is monitored by measuring serum creatinine, creatinine clearance, and proteinuria. These clinical markers are usually a late sign of renal damage and indicate its dysfunction [6]. What is more, these markers do not always correlate well with the severity of renal damage seen on biopsy [27]. The early stages of renal functions impairment are diagnosed only by measuring GFR. The complications of chronic renal disease increase with decreasing GFR [28]. There are a number of studies confirming the huge impact of GFR level in progression of renal damage [2932]. However, a good biomarker of decreased GFR, together with a proper marker of tubular injury, would allow for the diagnosis of renal failure in diabetic patients before increased albuminuria and irreversible kidney damage [13].

One of the specific renal proteins-uromodulin-has been found as urinary biomarker which positively correlates with GFR ratio and decreased uromodulin concentrations have been found in renal failure and diabetic nephropathy $[33,34]$. In our study, we observed that uromodulin is a 


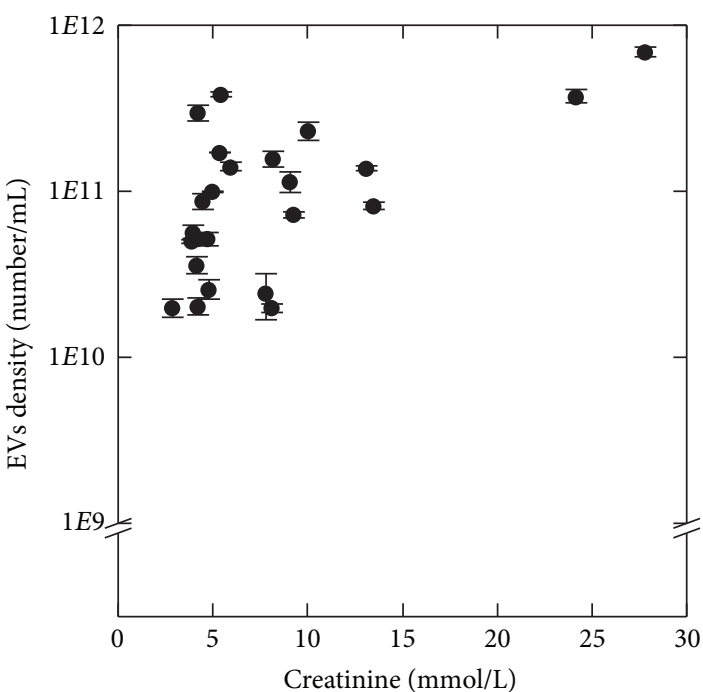

(a)

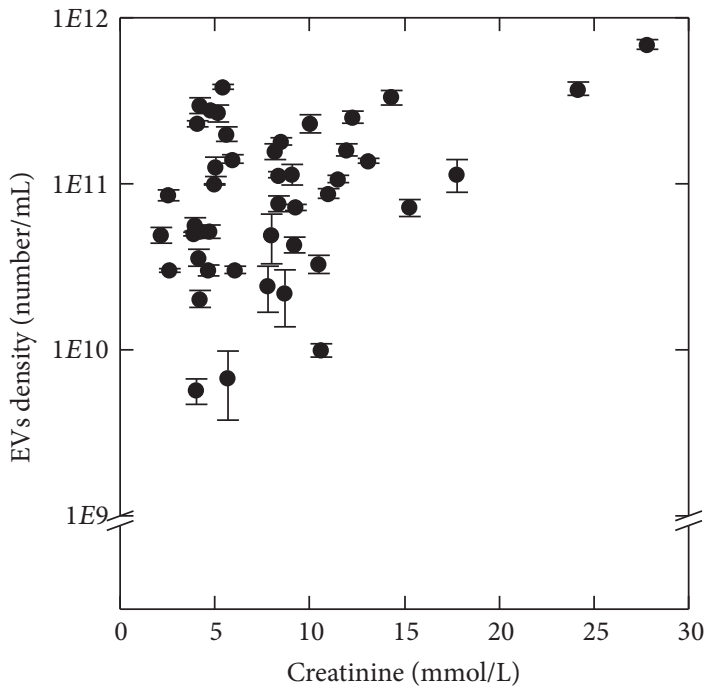

(c)

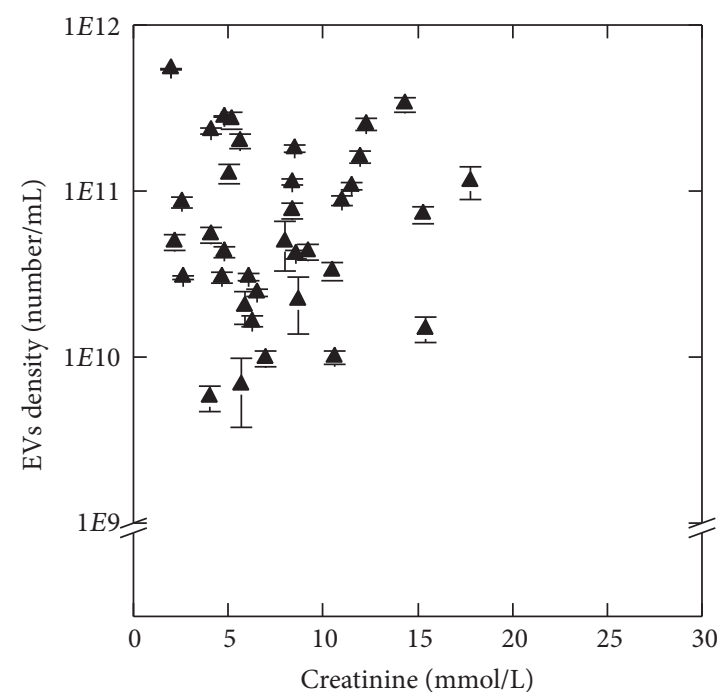

(b)

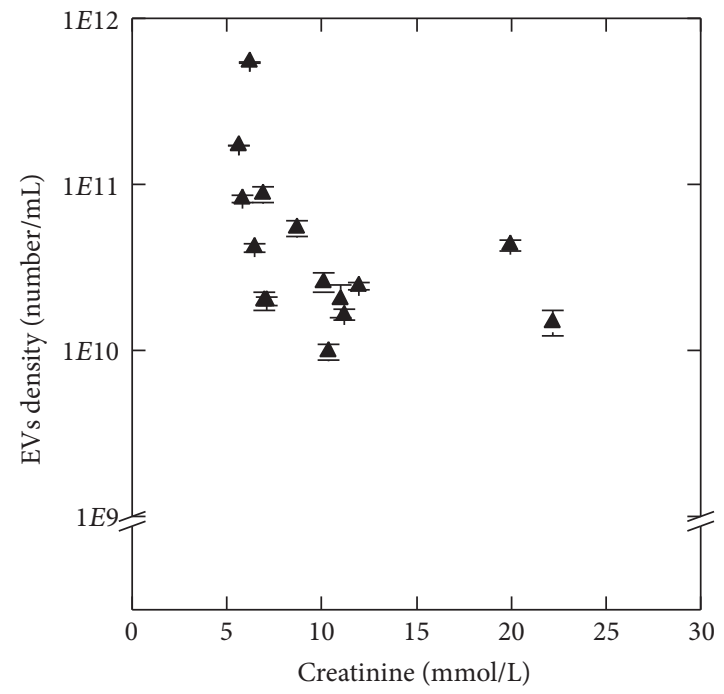

(d)

FIGURE 3: Relationship between EVs density and urine creatinine concentration in study groups: CD (a), UD (b), NRF (c), and RF (d). EVs density values are given as mean (SD). Spearman's rank correlation coefficient, $p<0.05$.

prominent protein related to EVs and we may assume that its drop in urine concentrations is related to decreased EV density in patient with renal failure. Uromodulin is probably involved in EVs clustering and precipitation (Figure 1(c)) [35].

In our study, we observed a negative correlation between EVs density and serum glucose level in RF and a negative tendency between these parameters in UD. Mehta [36] showed that the kidney is intimately involved in the development of hyperglycemia in the critically ill patients. Sechi et al. [37] demonstrated that abnormal plasma glucose levels were elevated when the GFR was $<50 \mathrm{~mL} / \mathrm{min} / 1.73 \mathrm{~m}^{2}$ and overall glucose metabolism parameters were not correlated with microalbuminuria (MA). These data are consistent with our study. We did not observe the correlation between MA and the EVs density in both $\mathrm{CD}$ and UD, as well as in RF and NRF. Thus we may assume that EVs presence is more related with impaired glucose metabolism and then with the presence of renal damage biomarker (MA) and EVs can be treated as the more ominous label of disease in diabetic patients. Interestingly, we also observed a positive correlation between EVs density and urine creatinine concentration in $\mathrm{NRF}$ and $\mathrm{CD}$, in contrast to those with more advanced disease stage (RF) or impropriate treatment (UD). This observation may suggest that the early renal dysfunction processes are more considerable in the urine EVs release. In the milder stage of renal failure we may expect the higher number of EVs in urine, as the primary marker of the renal dysfunction. In further study the more specific attention should be focused on the correlation between cystatin C and even angiopoietin 2, which appeared to be a relevant predictor of renal dysfunction in acute pancreatitis patients 


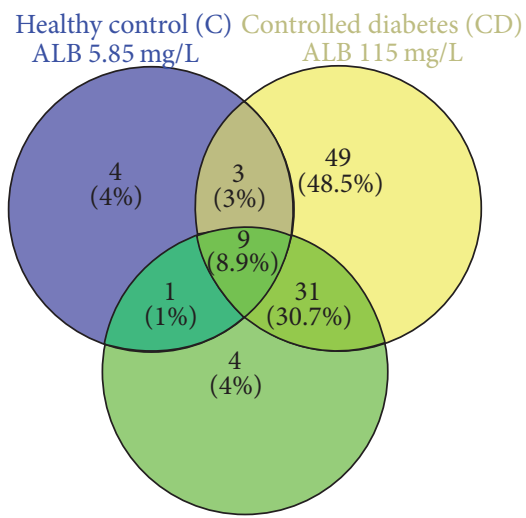

Uncontrolled diabetes (UD) ALB $6410 \mathrm{mg} / \mathrm{L}$

(a)

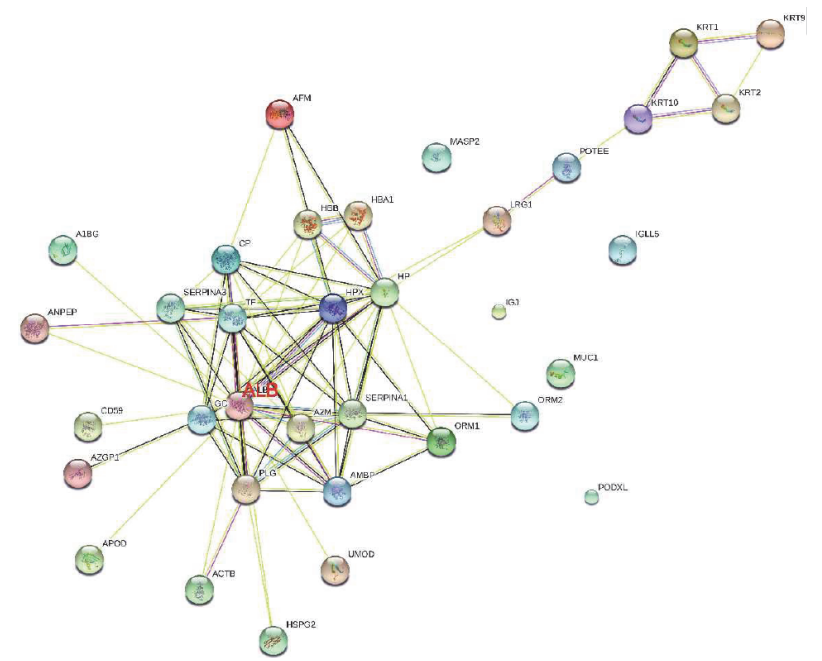

(b)

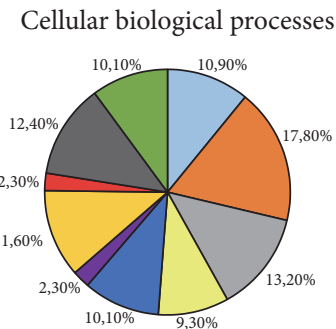

Molecular function

Cellular components
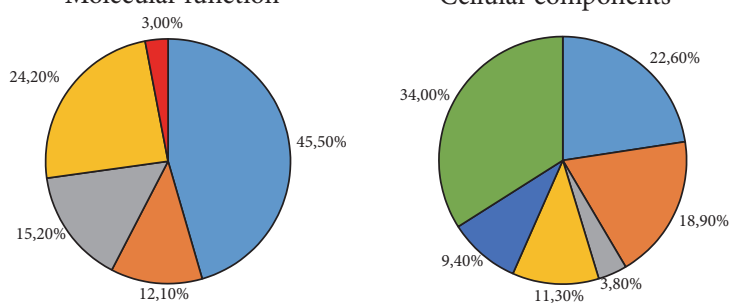

- Cellular component organization

- Binding

- Receptor activity

- Structural molecule activity

- Catalytic activity

- Transporter activity

ธ Biological regulation

- Response to stimulus

- Developmental process

- Multicellular organismal process

- Biological adhesion
- Membrane

- Macromolecular complex

- Extracellular matrix

口 Cell part

- Organelle

- Extracellular region

(c)

FIGURE 4: Continued. 


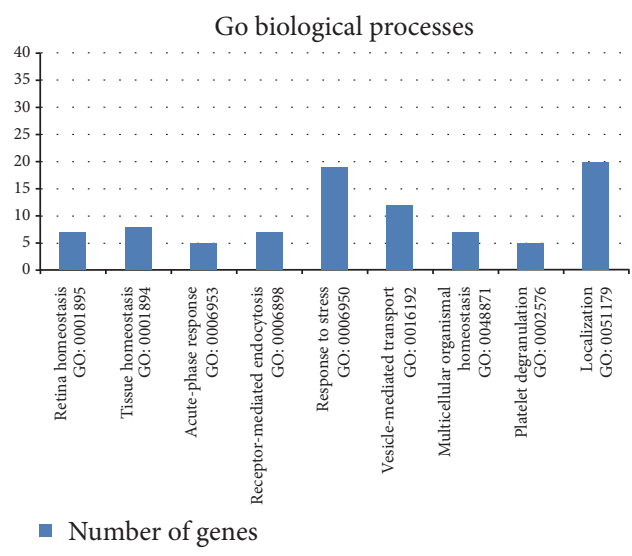

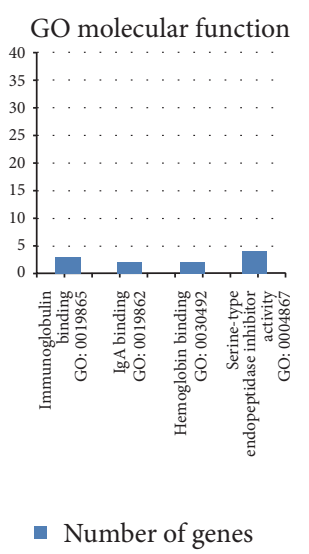

(d)

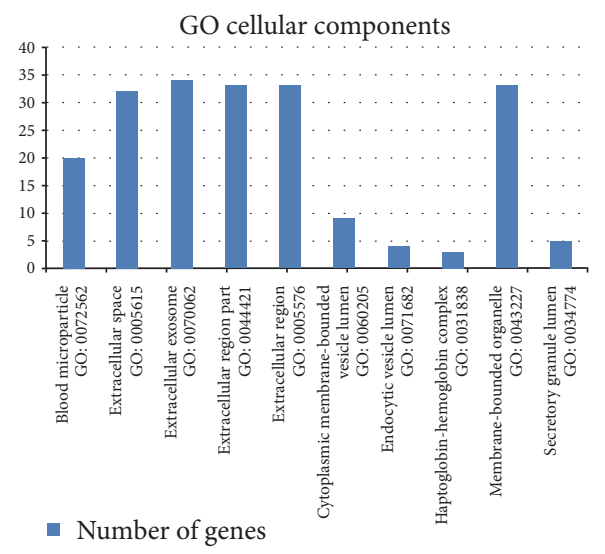

- Number of genes

FIGURE 4: Proteomic analysis of urinary extracellular vesicles: mass spectrometry results from representative samples. (a) Venn diagram shows that in EVs from controlled diabetic patient with microalbuminuria there is a large number of unique proteins $(n=49)$, in a healthy control and in an uncontrolled diabetic patient with macroalbuminuria the number of unique proteins is very low $(n=4)$ [20]. (b) Proteinto-protein interaction analysis of common 45 proteins selected from Venn diagram shows the central role of albumin among urinary EVrelated proteins; the list of submitted proteins is available in a supplementary data file (Supplementary Table 2) [22]. (c, d) Gene Ontology analysis showed that most of identified proteins are related to extracellular region or they are related to membrane organelles (exosomes); their localization corresponds with molecular function (receptors and transport proteins) [19]. A1BG: alpha-1-B glycoprotein; A2M: alpha-2-macroglobulin; ACTB: actin, beta; AFM: afamin; ALB: albumin; AMBP: alpha-1-microglobulin/bikunin precursor; ANPEP: alanyl (membrane) aminopeptidase; APOD: apolipoprotein D; AZGP1: alpha-2-glycoprotein 1, zinc-binding; CD59: CD59 molecule, complement; regulatory protein; CP: ceruloplasmin; GC: group-specific component (vitamin D binding protein); HBA1: hemoglobin, alpha 1; HBB: hemoglobin, beta; HP: haptoglobin; HPX: hemopexin; HSPG2: heparan sulfate proteoglycan 2; IGJ: immunoglobulin J polypeptide; IGLL5: immunoglobulin lambda-like polypeptide 5; KRT1: keratin 1; KRT2: keratin 2; KRT9: keratin 9; KRT10: keratin 10; LRG1: leucine-rich alpha2-glycoprotein 1; MASP2: mannan-binding lectin serine peptidase 2; MUC1: mucin 1; ORM1: orosomucoid 1; ORM2: orosomucoid 2; PLG: plasminogen; PODXL: podocalyxin-like; POTEE: POTE ankyrin domain family member E; SERPINA1: serpin peptidase inhibitor, clade A, member 1; SERPINA3: serpin peptidase; inhibitor, clade A, member 3; TF: transferrin; UMOD: uromodulin.

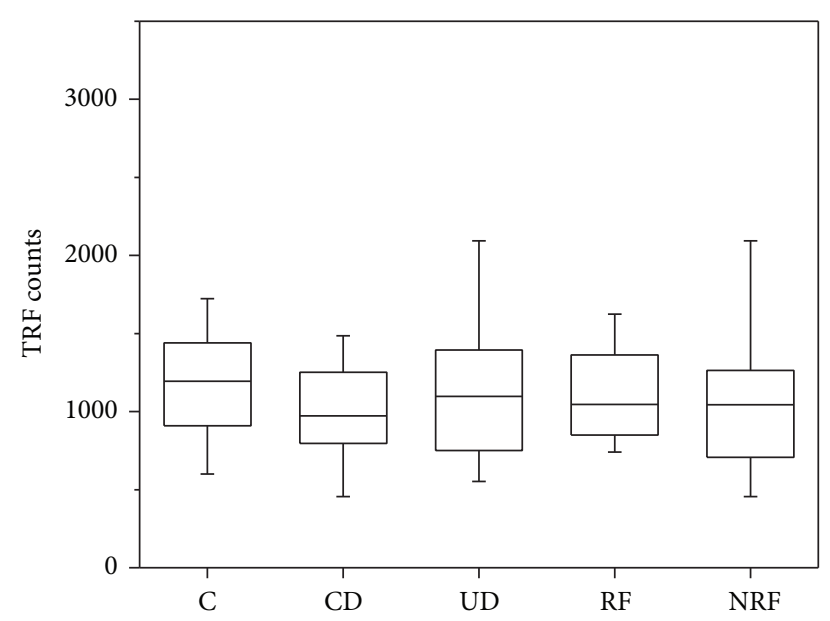

FIGURE 5: Urine CD81 level. Results from Time Resolved Fluorescence assay. Kruskal-Wallis test: median TRF counts, $p=0.5$ at $\alpha<$ 0.05 significance level.

[38]. Age is the strongest factor influencing physiological state, as well as renal function. According to the number of epidemiological studies, GFR declines with age average about $0.8 \mathrm{~mL} / \mathrm{min} / 1.73 \mathrm{~m}^{2}$ per year. The multiple regression (backward stepwise regression) model did not show the significant impact of age on changes in the amount of EVs.
Urinary EVs are enriched in membrane and cytosolic cargo proteins from the different epithelial cells lining the urinary track. To date, there are only few studies to reveal the new urine biomarkers, which are based on proteomic methods. Among them, proteases and protease inhibitors including kallikreins $[6,10,14]$ and metalloproteinases (MMP-2, MMP-7, and MMP-8) have been observed in normoalbuminuria and microalbuminuria groups. In macroalbuminuric patients, cathepsin $\mathrm{D}$ has been more abundant than in other patients [14]. In our study we found new protease inhibitors including inter-alpha-trypsin inhibitor (AMBPHuman), inhibitor of the complement membrane attack complex (CD59) and proteases including mannan-binding lectin serine protease 2 (MASP2_Human). A large number of unique proteins in controlled diabetes can be used in selection of potential biomarkers in further studies on the risk of diabetic nephropathy in such patients.

According to our observations the specific marker for exosomes (CD81) did not show significant difference in the level (Figure 5). The CD81 is a surface exosome marker which belongs to the tetraspanin family (TAPA-1) and is involved in signal transduction and cell adhesion [39]. CD81 protein is enriched in the exosome membrane [40]. However, it has not been shown if this biomolecule distinguish the severity of renal disease in diabetic patients. We may also speculate that in our study we did not observe significant differences in CD81 level between groups because the most numerous was 
CD81-negative (nonexosomes) population of microvesicles, membrane bubbles above $100 \mathrm{~nm}$ (Figures 1(e) and 1(f)).

In our study we confirmed that the quantitative analysis of urinary EVs seems to be a promising tool for defining new biomarkers [41]. We pictured that EVs are present in urine and they maintain their integrity during sampling and preparation process. However, most of these studies are focused on the two areas of progress: bladder or prostatic cancer and acute rejection of renal transplant [10]. Very recent study on diabetic nephropathy novel biomarkers revealed that exosomal regucalcin was underexpressed in renal disease patients [42].

\section{Conclusions}

Finally we may conclude that urinary EVs have the potential to be biomarkers of renal damage in diabetic patients, and the number of structural and enzymatic proteins (including uromodulin) can be found in urine EVs fraction to be in use as their indicators or new biomarkers both of renal failure and of diabetic nephropathy in the future. The easy accessibility of EVs in urine can increase their use as biomarkers compared to invasive biopsy. Further validation, characterization of the content of bioactive molecules, and larger study population are needed.

\section{Abbreviations}

CD: Properly controlled diabetic patients

DMt1: Type 1 diabetes mellitus

DMt2: Type 2 diabetes mellitus

EDTA: Ethylenediaminetetraacetic acid

ESEM: Environmental Scanning Electron Microscopy

EVs: Extracellular vesicles

GFR: Glomerular Filtration Rate

GO: Gene Ontology

HbAlc: Glycated hemoglobin

MA: Microalbuminuria

NRF: Diabetic patients without renal failure

PBS: Phosphate-buffered saline

RF: Diabetic patients with renal failure

TEM: Transmission Electron Microscopy

TRPS: Tunable Resistive Pulse Sensing

UD: Poorly controlled diabetic patients.

\section{Competing Interests}

The authors declare that they have no competing interests.

\section{Acknowledgments}

This study was supported by the Polish National Science Centre (NCN) the Grant OPUS 4 for Ewa Stępień (2012/07B/NZ5/02510) and the Polish Ministry of Science and Higher Education (MNSW), the grant for Agnieszka Kamińska (7150/E-338/M/2015). The research was carried out with equipment purchased with financial support from the European Regional Development Fund in the framework of the Polish Innovation Economy Operational Program
(Contract no. POIG.02.01.00-12-023/08). The authors would like to thank also Aleksandra Tokarz for providing clinical database of patients.

\section{References}

[1] K. R. Tuttle, G. L. Bakris, R. W. Bilous et al., "Diabetic kidney disease: a report from an ADA consensus conference," Diabetes Care, vol. 37, no. 10, pp. 2864-2883, 2014.

[2] D. J. Leehey, H. J. Kramer, T. M. Daoud, M. P. Chatha, and M. A. Isreb, "Progression of kidney disease in type 2 diabetesbeyond blood pressure control: an observational study," $B M C$ Nephrology, vol. 6, pp. 1471-2369, 2005.

[3] National Kidney Foundation, Diabetes-a major risk factor for kidney disease, https://www.kidney.org/news/newsroom/factsheets/FastFacts/.

[4] Y. Yuana, A. Sturk, and R. Nieuwland, "Extracellular vesicles in physiological and pathological conditions," Blood Reviews, vol. 27, no. 1, pp. 31-39, 2013.

[5] A. Ranghino, V. Dimuccio, E. Papadimitriou, and B. Bussolati, "Extracellular vesicles in the urine: markers and mediators of tissue damage and regeneration," Clinical Kidney Journal, vol. 8, no. 1, pp. 23-30, 2015.

[6] A. Gámez-Valero, S. I. Lozano-Ramos, I. Bancu, R. LauzuricaValdemoros, and F. E. Borràs, "Urinary extracellular vesicles as source of biomarkers in kidney diseases," Frontiers in Immunology, vol. 6, no. 6, Article ID 00006, 2015.

[7] D. A. Shifrin Jr., M. D. Beckler, R. J. Coffey, and M. J. Tyska, "Extracellular vesicles: communication, coercion, and conditioning," Molecular Biology of the Cell, vol. 24, no. 9, pp. 1253-1259, 2013.

[8] M. P. Zaborowski, L. Balaj, X. O. Breakefield, and C. P. Lai, "Extracellular vesicles: composition, biological relevance, and methods of study," BioScience, vol. 65, no. 8, pp. 783-797, 2015.

[9] L. Musante, D. E. Tataruch, and H. Holthofer, "Use and isolation of urinary exosomes as biomarkers for diabetic nephropathy," Frontiers in Endocrinology, vol. 5, article 149, 2014.

[10] T. Pisitkun, R. Johnstone, and M. A. Knepper, "Discovery of urinary biomarkers," Molecular and Cellular Proteomics, vol. 5, no. 10, pp. 1760-1771, 2006.

[11] E. A. Lock, "Sensitive and early markers of renal injury: where are we and what is the way forward?" Toxicological Sciences, vol. 116, no. 1, pp. 1-4, 2010.

[12] D. Levenson, "The search for improved markers of acute kidney injury," https://www.aacc.org/publications/cln/articles/2014/january/kidney-injury/.

[13] A. Żyłka, A. Gala-Błądzińska, K. Rybak, P. Dumnicka, R. Drożdż, and B. Kuśnierz-Cabala, "Role of new biomarkers for the diagnosis of nephropathy associated with diabetes type 2," Folia Medica Cracoviensia, vol. 55, no. 4, pp. 21-33, 2015.

[14] L. Musante, D. Tataruch, D. Gu et al., "Proteases and protease inhibitors of urinary extracellular vesicles in diabetic nephropathy," Journal of Diabetes Research, vol. 2015, Article ID 289734, 14 pages, 2015.

[15] F. A. Coumans, E. van der Pol, A. N. Böing et al., "Reproducible extracellular vesicle size and concentration determination with tunable resistive pulse sensing," Journal of Extracellular Vesicles, vol. 3, Article ID 25922, 2014.

[16] E. L. C. J. Blundell, L. J. Mayne, E. R. Billinge, and M. Platt, "Emergence of tunable resistive pulse sensing as a biosensor," Analytical Methods, vol. 7, no. 17, pp. 7055-7066, 2015. 
[17] E. L. C. J. Blundell, R. Vogel, and M. Platt, "Particle-by-particle charge analysis of dna-modified nanoparticles using tunable resistive pulse sensing," Langmuir, vol. 32, no. 4, pp. 1082-1090, 2016.

[18] W. Anderson, R. Lane, D. Korbie, and M. Trau, "Observations of tunable resistive pulse sensing for exosome analysis: improving system sensitivity and stability," Langmuir, vol. 31, no. 23, pp. 6577-6587, 2015.

[19] J. R. Wiśniewski and M. Mann, "Consecutive proteolytic digestion in an enzyme reactor increases depth of proteomic and phosphoproteomic analysis," Analytical Chemistry, vol. 84, no. 6, pp. 2631-2637, 2012.

[20] J. Kasprzyk, E. Stępień, and W. Piekoszewski, "Application of nano-LC-MALDI-TOF/TOF-MS for proteomic analysis of microvesicles," Clinical Biochemistry, 2016.

[21] P. D. Thomas, M. J. Campbell, A. Kejariwal et al., "PANTHER: a library of protein families and subfamilies indexed by function," Genome Research, vol. 13, no. 9, pp. 2129-2141, 2003.

[22] Venny 2.1 by Juan Carlos Oliveros, BioinfoGP, CNB-CSIC, http://bioinfogp.cnb.csic.es/tools/venny/.

[23] J. Bobrowska, J. Pabijan, J. Wiltowska-Zuber et al., "Protocol of single cells preparation for time of flight secondary ion mass spectrometry," Analytical Biochemistry, vol. 511, pp. 52-60, 2016.

[24] D. Szklarczyk, A. Franceschini, S. Wyder et al., "STRING v10: protein-protein interaction networks, integrated over the tree of life," Nucleic Acids Research, vol. 43, no. 1, pp. D447-D452, 2015.

[25] C. A. Rabito, F. Panico, R. Rubin, N. Tolkoff-Rubin, and R. Teplick, "Noninvasive, real-time monitoring of renal function during critical care," Journal of the American Society of Nephrology, vol. 4, no. 7, pp. 1421-1428, 1994.

[26] A. E. Turco, W. Lam, A. D. Rule et al., "Specific renal parenchymal-derived urinary extracellular vesicles identify age-associated structural changes in living donor kidneys," Journal of Extracellular Vesicles, vol. 5, 2016.

[27] P. McFarlane, R. E. Gilbert, L. MacCallum, and P. Senior, "Chronic kidney disease in diabetes," Canadian Journal of Diabetes, vol. 37, no. 1, pp. S129-S136, 2013.

[28] C. Thomas and L. Thomas, "Renal failure-measuring the glomerular filtration rate," Deutsches Ärzteblatt International, vol. 106, no. 51-52, pp. 849-854, 2009.

[29] W. Metcalfe, "How does early chronic kidney disease progress? A background paper prepared for the UK consensus conference on early chronic kidney disease," Nephrology Dialysis Transplantation, vol. 22, no. 9, pp. 26-30, 2007.

[30] P. Palatini, "Glomerular hyperfiltration: a marker of early renal damage in pre-diabetes and pre-hypertension," Nephrology Dialysis Transplantation, vol. 27, no. 5, pp. 1708-1714, 2012.

[31] B. M. Brenner, E. V. Lawler, and H. S. Mackenzie, "The hyperfiltration theory: a paradigm shift in nephrology," Kidney International, vol. 49, no. 6, pp. 1774-1777, 1996.

[32] V. Rigalleau, C. Lasseur, C. Perlemoine et al., "Estimation of glomerular filtration rate in diabetic subjects: cockcroft formula or modification of diet in renal disease equation?" Diabetes Care, vol. 28, no. 4, pp. 838-843, 2005.

[33] C. Thornley, A. Dawnay, and W. R. Cattell, "Human TammHorsfall glycoprotein: urinary and plasma levels in normal subjects and patients with renal disease determined by a fully validated radioimmunoassay," Clinical Science, vol. 68, no. 5, pp. 529-535, 1985.
[34] A. M. Bernard, A. A. Ouled, R. R. Lauwerys, A. Lambert, and B. Vandeleene, "Pronounced decrease of Tamm-Horsfall proteinuria in diabetics," Clinical Chemistry, vol. 33, no. 7, article $1264,1987$.

[35] T. F. Hiemstra, P. D. Charles, T. Gracia et al., "Human urinary exosomes as innate immune effectors," Journal of the American Society of Nephrology: JASN, vol. 25, no. 9, pp. 2017-2027, 2014.

[36] R. L. Mehta, "Glycemic control and critical illness: is the kidney involved?" Journal of the American Society of Nephrology, vol. 18, no. 10, pp. 2623-2627, 2007.

[37] L. A. Sechi, C. Catena, L. Zingaro, A. Melis, and S. De Marchi, "Abnormalities of glucose metabolism in patients with early renal failure," Diabetes, vol. 51, no. 4, pp. 1226-1232, 2002.

[38] M. Sporek, P. Dumnicka, A. Gala-Błądzińska et al., "Angiopoietin-2 is an early indicator of acute pancreaticrenal syndrome in patients with acute pancreatitis," Mediators of Inflammation, vol. 2016, Article ID 5780903, 7 pages, 2016.

[39] S. Levy, S. C. Todd, and H. T. Maecker, "CD81 (TAPA-1): a molecule involved in signal transduction and cell adhesion in the immune system," Annual Review of Immunology, vol. 16, pp. 89-109, 1998.

[40] D. Perez-Hernandez, C. Gutiérrez-Vázquez, I. Jorge et al., "The intracellular interactome of tetraspanin-enriched microdomains reveals their function as sorting machineries toward exosomes," Journal of Biological Chemistry, vol. 288, no. 17, pp. 11649-11661, 2013.

[41] G. Pocsfalvi, D. A. A. Raj, I. Fiume, A. Vilasi, F. Trepiccione, and G. Capasso, "Urinary extracellular vesicles as reservoirs of altered proteins during the pathogenesis of polycystic kidney disease," Proteomics-Clinical Applications, vol. 9, no. 5-6, pp. 552-567, 2015.

[42] I. Zubiri, M. Posada-Ayala, A. Benito-Martin et al., "Kidney tissue proteomics reveals regucalcin downregulation in response to diabetic nephropathy with reflection in urinary exosomes," Translational Research, vol. 166, no. 5, pp. 474-484.e4, 2015. 


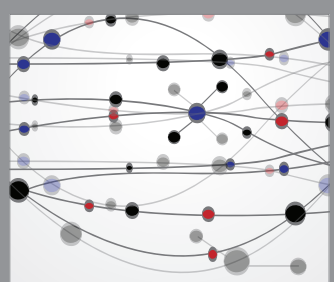

The Scientific World Journal
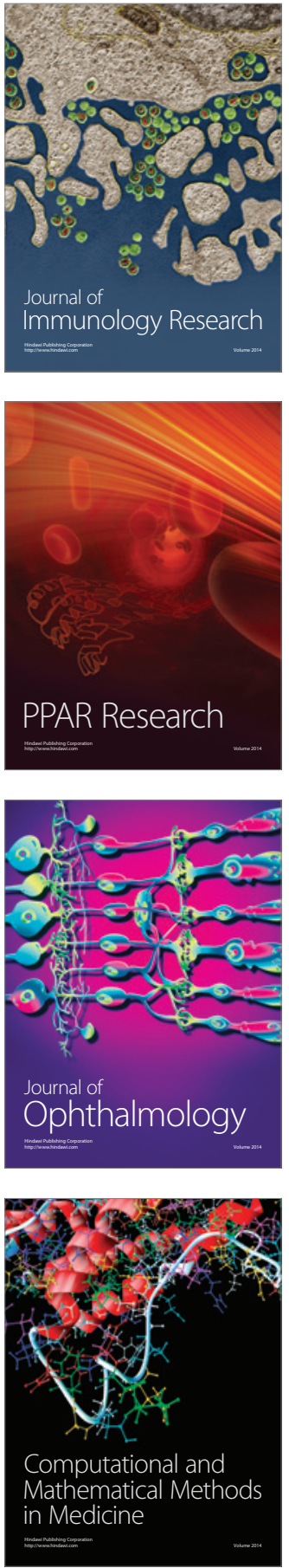

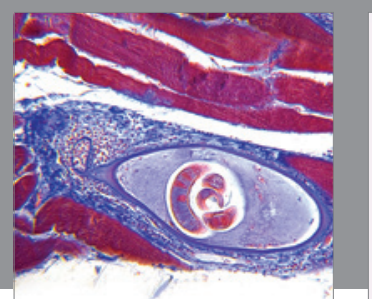

Gastroenterology Research and Practice

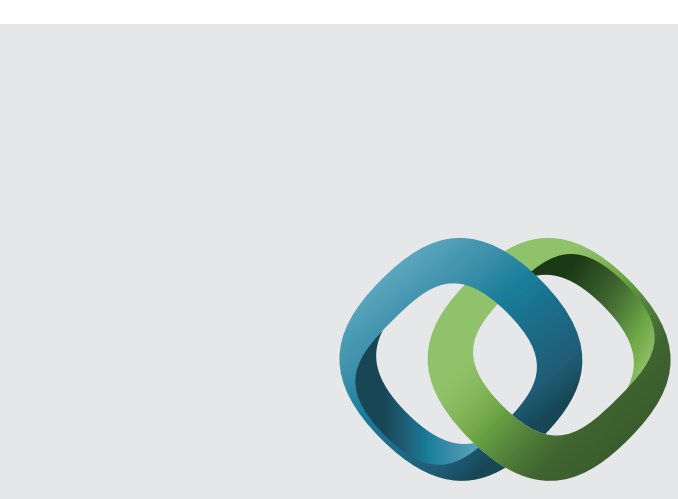

\section{Hindawi}

Submit your manuscripts at

http://www.hindawi.com
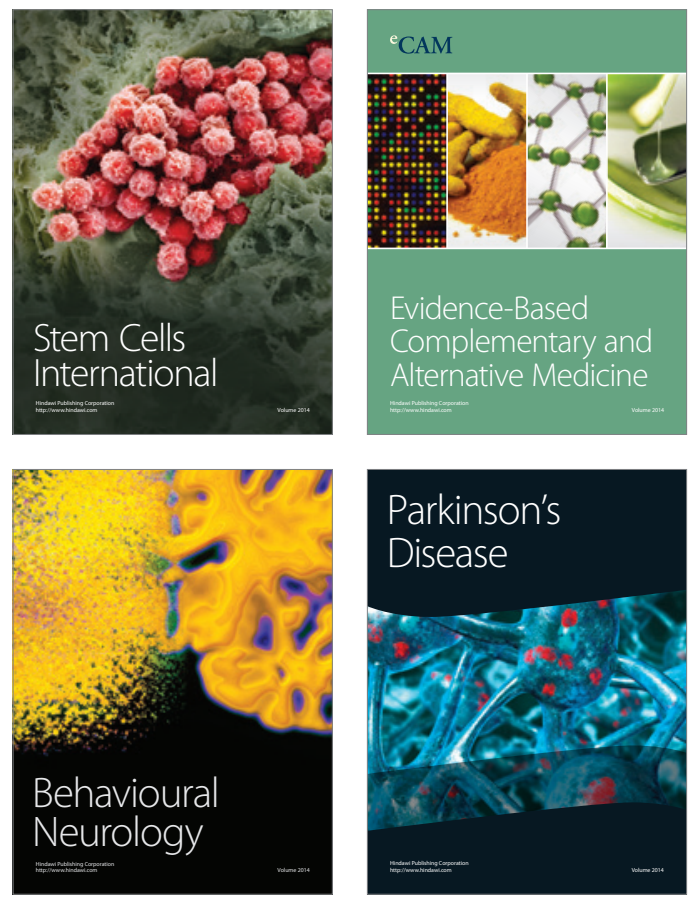
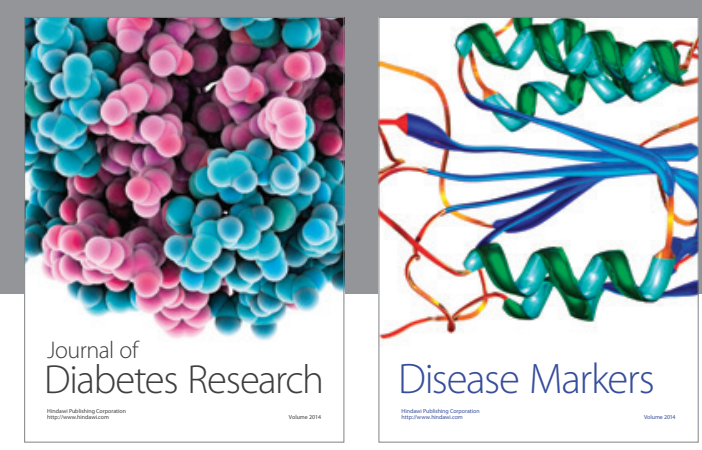

Disease Markers
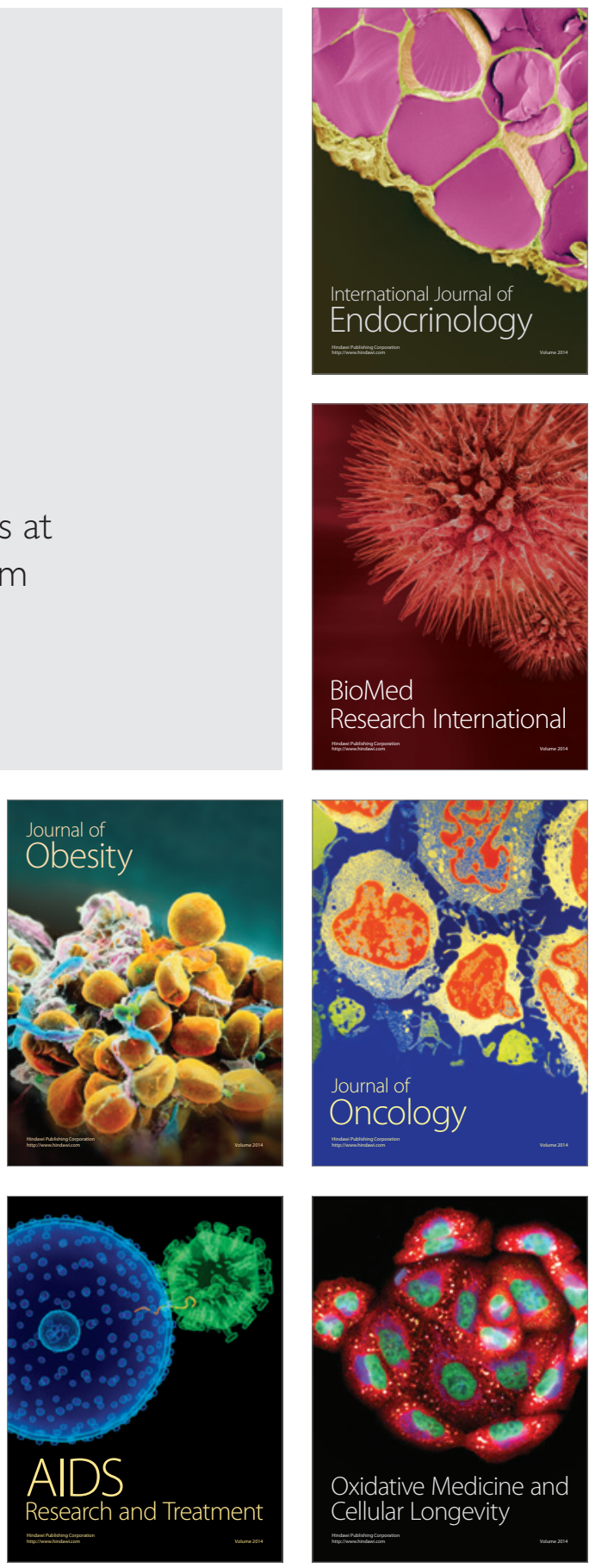